\title{
Reluctant empiricists: community mental health nurses and the art of evidence-based praxis
}

Paul Crawford ${ }^{1}$ RMN DPSN BA(Hons) PhD,

Brian Brown ${ }^{2} \mathrm{BSc}(\mathrm{Hons}) \mathrm{PhD}$,

Paul Anthony ${ }^{3}$ RMN MSc and

Carolyn Hicks ${ }^{4}$ BA MA PhD CPsychol

${ }^{1}$ School of Nursing, Queens Medical Centre, University of Nottingham, Nottingham, UK, ${ }^{2}$ Faculty of Health and Community Studies, De Montfort University, Leicester, UK, ${ }^{3}$ The Foundation NHS Trust, Stafford and School of Health, Staffordshire University, Stafford, UK and ${ }^{4}$ Department of Health Sciences, University of Birmingham, Birmingham, UK

Correspondence:

Dr Brian Brown Division of Psychology and Speech and Language Therapy, De Montfort University, Scraptoft Campus, Scraptoft, Leicester, LE7 9SU UK E-mail: brown@dmu.ac.uk

Health and Social Care in the Community 10(4), 287-298, 2002

\section{Abstract}

The National Service Framework for Mental Health (1999) emphasizes the need for a culture of evidence-based practice (EBP) in mental health care. However, there is relatively little research addressing EBP from the perspective of community mental health nurses and we are still unsure of why the uptake of this style of working has been slow. This paper suggests that rather than thinking in terms of 'barriers' to the uptake of EBP, the issue may best be conceptualized as a form of praxis on the part of nurses, as they seek to manage the diversity of ideologies and practices in their working lives. From an interview and focus group study, we identify how practitioners' narrow definition of EBP itself, their formulation of how EBP was at odds with the nurse's professional activity and the organizational constraints within which they work were perceived to inhibit access to information and offer little time and managerial support for information seeking. Those who attempt to further the involvement of community mental health staff in EBP will have to reconceptualize the reasons why staff have yet to incorporate it fully, and acknowledge that this does not occur because staff are simply 'ignorant Luddites', but that this resistance enables them to retain a sense of control over their working lives and retain a focus on work with clients. Future EBP initiatives will have to address these ideological and organizational factors in order for uptake to be accelerated. This may involve changing organizational cultures and work roles and even encouraging activism on the part of the practitioners so as to enable them to learn from each other and educate and change their work environments. 
Keywords: community mental health nursing, evidence-based practice, research

The concept of evidence-based practice (EBP) is currently yielding a great deal of coverage in the healthcare literature (Closs \& Cheater 1999, Dinsdale 2000, Jackson 1999, Clarke 2000, McKenna et al. 2000, Nieboer et al. 1999a, 1999b; McMillan 2000). It is also a national priority within the UK's NHS (Department of Health 1999a, 1999b), being an integral component of the National Service Framework for Mental Health and a cornerstone of clinical governance. In the view of its advocates and policy makers, the adoption of EBP contributes to the goal of providing high-quality, clinically effective mental health care (Brooker 2000; Department of Health 1999a, 1999b; McMillan, 2000).

Yet despite this enthusiasm, a number of important contradictions exist and crucial questions remain unanswered. Defining EBP, identifying the barriers to its implementation and characterizing the changes that are needed to optimize the use of evidence by healthcare practitioners are all areas that yield a variety of competing viewpoints. Allied to this, as we shall see, there is a suspicion that some sectors of the healthcare workforce are not climbing aboard the bandwagon as rapidly as policy makers might have hoped.

It is our purpose here to begin to delineate what it is that community practitioners are doing with regard to EBP. We shall focus on some of the reasons why staff in community mental health nursing might be having difficulty in achieving the goals of EBP. It is our contention that nurses are not merely lagging behind the new spirit of EBP, but are actively engaging in a form of praxis - managing occupational diversity and balancing the demands of clients, therapeutic approaches and the interpersonal processes of nursing - and EBP is being slotted in to this diverse mosaic of practice. We have adopted the term praxis because of its connotations of meaningful decision making and activity, and it is our intention to begin to characterize what form this might take.

The debate over EBP has so far been dominated by the conflict between enthusiasts for EBP and those who feel that EBP does not capture the important aspects of the nursing process; there has been little systematic attempt to map out and theorize how nurses themselves are actively managing diversity as they confront the competing and often contradictory imperatives of their organizational context, their professional identity and their caring work.

\section{Diversity in definition: what is evidence-based practice?}

Evidence-based practice is not always a monolithic regime of enlightenment rationality. Even the definition of EBP is subject to some debate in the literature and there is considerable uncertainty as to what it might include. One widely quoted working definition is provided by Sackett et al. (1997), who define EBP as:

The conscientious, explicit and judicious use of current best evidence in making decisions about the care of individual patients, based on skills which allow the doctor ( $s i c$ ) to evaluate both personal experience and external evidence in a systematic and objective manner. (p. 71) 
Whereas definitions like this emphasize both the personal experience of the clinician as well as formal research evidence, a much less plural definition of the kinds of evidence that are legitimate is found in the UK's National Service Framework for Mental Health (Department of Health 1999a, p. 9), which presents a hierarchy of evidence it sees to be applicable, with the randomized controlled trial or better still a systematic review of several trials - sitting at the top of the list. The inclusion of opinion and experience is admissible at the lower end of its hierarchy, but even here it is restricted to the opinion and experience of 'experts'. At the same time, some authors have argued that there are important aspects of the nursing process that are not compatible with the evidence-based paradigm, for example that the 'participative nurse -person processes' (Mitchell 1997, p. 154) are depleted by a model of care that encourages the nurse to generalize or standardize care for all patients in a category rather than encouraging 'individualized care that respects the wishes and choices of the people served' (Mitchell 1997, p. 155; Lines 2001, p. 170).

Thus, so far, much debate over EBP has arisen from some theorists seeking greater recognition for opinion, clinical experience and individualized, recipient-tailored approaches to care in the face of the standardization that they believe is advocated under EBP. However, this kind of debate has so far been conducted in the pages of journals and this in itself does not necessarily capture the picture of practice as community mental health staff negotiate the competing demands of practical nursing work.

\section{Barriers to the implementation of EBP}

In theory at least, the arguments in favour of EBP are persuasive on scientific, humane and economic grounds. The spectre of expensive and ineffective routines and customs falling to the astute gaze of empirical scrutiny is an attractive one (Ghali et al. 1999). Given the support on the part of researchers, policy makers and managers for this approach to health care, it is perhaps important to understand why it is not progressing as rapidly as some enthusiasts would like among healthcare staff (Traynor 1999). In mental health in particular, there are many commentators who complain of an enduring theory-practice gap (Reynolds 2000, Upton 1999).

In this paper, then, we will consider some of the reasons why progress may not be so rapid as enthusiasts would hope. We shall be aided by a selection of evidence from a study of the attitudes of community mental health nurses $(\mathrm{CMHNs})$ to EBP. This particular case is of special interest because of two major factors that have a bearing on whether EBP can be achieved.

Firstly, there is a notorious difficulty in applying the formal models of EBP to mental health care, and nursing in particular, because no matter how fully the details of a psychological intervention are specified, there will be aspects of the way it is delivered in practice that may vary in clinically significant ways, over which the researcher has no control (Parry 2000).

Secondly, staff working in community settings might have particular difficulties with accessing evidence. Much promotion of EBP has centred on initiatives in teaching hospitals; these are directed 
at doctors, whose training curricula have already familiarized them with quantitative research methods (Bilsker \& Gouldner 1999, French 1999). Therefore, it would be useful to examine how far it can be achieved in settings where library facilities are not on site and the practitioners' parent organizations do not subscribe to electronic databases to facilitate literature searching. It is in these 'swampy lowlands' (Schon 1987) that a great deal of health care is undertaken and it is in these heartlands of care that EBP must be viable if it is to become a flourishing practice model.

Whereas opportunities to discuss EBP in mental health as a whole are developing, especially with the foundation of 'Evidence-based Mental Health' in 1998, there is still relatively little material that relates to mental health nursing (Brooker et al. 1996). The difficulties experienced by would-be researchers in this field may reflect a more fundamental ambivalence about the philosophy and purpose of the discipline (Forchuk 2001). This is compounded by complaints of a lack of high-quality research suitable for application in practice (Holloway 2000, Veeramah 1995, Wilson-Barnett et al. 1990) or, conversely, the overabundance of often conflicting research (Hunt 1987).

The conventional approach to making the desire of policy makers, managers and EBP enthusiasts a reality is to focus on the individual practitioners, especially those who are still in training. Within this approach, the most popular solution seems to have been to change the attitudes and enhance the evidence-collecting and reviewing skills of practitioners. Whereas there are some initiatives to develop nurses as researchers (French 1999), a great many of the schemes to teach evidence-based medicine have so far focused on doctors rather than nurses (Bilsker \& Gouldner 1999). This assumption that we can change the culture of the healthcare professions by educating practitioners has led to much pedagogic innovation, but progress is still slow when it comes to embedding evidence-based health care in practice. It is our intention, then, to examine what might be holding back mental health nursing vis-à-vis evidence-based health care.

There have also been some more theoretically critical attempts to shed light on why there appears to be an enduring gap between practitioners' everyday life worlds and the theoretical and scientific knowledge in nursing (Traynor 1999, 2000). Here, the exhortations from policy makers are seen as a kind of social control exerted over nurses, who may lack a robust intellectual tradition of their own (Veeramah 1995) and have an unhappy history of subservient, non-academic status that may conspire to prevent the application of evidence in practice (Grant \& Mills 2000, Walsh \& Ford 1989). Moreover, some have argued that there is far more to the interpersonal process of performing the role of a nurse than can ever be captured within evidence-based protocols (Barker 2001, Flaming 2001). Equally, there are attempts to address the difficulties of implementing EBP using questionnaires (Parahoo 2000, Dunn et al. 1998) that have identified factors relating to the individuals themselves and their organizations as constraints on the adoption process. The major limitation of questionnaire surveys is that they treat the respondents as 'honest souls' who wear their hearts on their sleeves (Potter \& Wetherell 1987) and who will present their feelings transparently in response to the questions. This approach also assumes that attitudes, perceptions of barriers to progress and awareness of constraints can all be treated as if they were measurable entities that could be 
represented as numbers on a scale.

\section{The study}

We have attempted here to adopt a more qualitative approach that is sensitive to the 'situated logics in use' (Hawes 1977) of the practitioners. Therefore, in this paper we attempt a more sensitive evocation of the logically connected narratives developed by practitioners as they describe what EBP means, and as they identify some of the constraints on its development in their work situation. Rather than reflecting the 'honest souls' of practitioners, these accounts are strategic formulations of the issues confronting them and the interviewers as they work together to establish the meaning of their circumstances. The clues as to why EBP is difficult to accomplish will be found not in measures of reliability but in a fine-grained search for contradiction, inconsistency and the use of language to construct a plausible version of events.

\section{Method and participants}

\section{Research design}

The exploratory nature of the study, coupled with the need to capture the unique perceptions of CMHNs, necessitated a qualitative research approach using semi-structured interviews and a focus group to facilitate discussion and debate and perhaps yield data inaccessible via one-to-one interviewing (Cormack 1996, Morgan \& Krueger 1997). The use of two methods of data collection was a kind of 'methodological triangulation' to enhance the study's validity (Polit \& Hungler 1995). Focus groups may be dominated by a few articulate participants (Morgan \& Krueger 1998), so individual interviews ensured that a range of individual perceptions could be gathered.

\section{The questions}

The same set of questions was used in both the focus group and the individual interviews. Topics for discussion included:

1 What does the concept of EBP mean to you?

2 To what extent is EBP a reality within community mental health nursing?

3 What are the inhibiting factors or barriers to EBP?

4 What value do you place on the concept of EBP?

5 What are the main priority areas for the development of EBP?

6 What resources or support is needed to promote and develop EBP within community mental health nursing?

The questions were formulated to elicit the views of $\mathrm{CMHNs}$ in relation to the main themes emerging from a critique of the literature in relation to EBP. The first question was intended to discern CMHNs' perspective in relation to the debate over definitions of EBP (McKenna et al. 2000, Coyler \& Kamath 1999). The second, third and fourth questions were intended to elicit a response in relation to 
emergent allegations in the literature that many mental health nurses may not adopt an EBP approach (Gournay 1995, Grant \& Mills 2000) and that in general many factors can conspire to inhibit EBP (Berk \& Leigh Janet 1999, Bonell 1999, Closs \& Cheater 1999, Ray 1999, Retsas 2000 , Veeramah 1995). The remaining questions were intended to ascertain if strategies seen as promoting EBP in other disciplines and contexts (NHS Centre for Reviews and Dissemination 1999, Pearcey 1995, Retsas 2000) would be seen as viable by CMHNs.

\section{Research participants}

The following criteria were used in identifying research participants:

RMN or equivalent qualification;

at least 1 year working as a generic community mental health nurse; and currently fit for and attending work.

The inclusion criteria ensured that participants would be in a position to make informed comments on the topics. Generic CMHNs were targeted because of their experience of community mental health nursing with a diverse range of client groups within a range of healthcare settings.

A sampling frame including members of all the eligible community mental health teams enabled interview participants to be sampled at random. CMHNs from one community mental health team who were not also participating in the individual interviews were invited to participate in the focus group.

\section{The focus group}

In line with methodological recommendations (Morgan \& Krueger 1997), a focus group comprising six $\mathrm{CMHNs}$ from one generic community mental health team was assembled. The participants knew each other well, and this helped to ensure that their contributions were less affected by social desirability bias (Cormack 1996). The group members comprised two ' $G$ ' grade and four ' $E$ ' grade staff, of whom the majority (five) were women. They ranged in age from 25 to 50 and they had undertaken varied lengths of service in community mental health, with an average of approximately 6 years, though two had served just over 15 years. Three of the focus group members had previously worked in an acute hospital setting and four had undertaken some EBP training after having qualified.

The meeting lasted for 90 minutes and was facilitated by the researcher, who 'actively facilitated' the meeting and ensured that the discussion remained focused and relevant. The participants were invited to share their responses to the questions within the group and a lively discussion ensued to clarify meanings and assess the responses. The emergent themes for each question were collated and recorded on a flip chart.

\section{The individual interviews}


Ten interviews were conducted with generic CMHNs comprising six 'G' grade and four 'E' grade nurses, of whom the majority (seven) were women. This group had undertaken an average of 4 years service in community mental health and four of them had undertaken some training specifically to familiarize themselves with EBP. The interviews were tape-recorded and transcribed.

\section{Analysis}

The transcripts were then subjected to thematic analysis utilizing Colaizzi's (1978) procedural phases. This involved repeatedly reading interview transcripts to gain a 'holistic' grasp of the issues and then extracting significant statements relating to EBP and the barriers inhibiting it. Meanings were formulated from the significant statements and phrases and these were then organized into themes.

Strategies to ensure the methodological integrity of the study included checking for internal consistency, method triangulation by means of comparing the individual and focus group interviews, participant validation, peer review and active reflection on the research process. Internal consistency was assessed by judging the 'equivalence' of responses within each interview (Slevin \& Sines 2000). This involved keeping brief field notes during the interview and jotting down key points made by the interviewee during the interview. An ongoing check could then be made for responses that were not in agreement with what the respondent had previously stated. There was no inconsistency noted during the interviews.

Participant validation was achieved by ensuring some of the original respondents from the interviews had the opportunity to comment on the data and findings; they were supplied with a copy of their transcript and subsequent theme construction and were asked to comment on the accuracy of analysis. Concurrent validation was achieved in the focus group through discussion and clarification of themes. Furthermore, in addition to the researchers, a further inspection of the data and analysis was undertaken by a nurse colleague, who traced the process of theme construction from the transcripts and ultimately agreed with the analysis. Finally, a reflective process using a research diary and field notes made during the interviews and immediately after the focus group was used to reduce the potential for researcher values, beliefs and preconceptions to influence the subsequent findings (Polit \& Hungler 1995).

\section{Ethical considerations}

An information sheet, outlining the design and purpose of the study, was provided for respondents to ensure informed consent and assure them that participation was voluntary. It was also stressed that participants could withdraw from the study at any stage and also withdraw any information they had provided during the study.

Potential respondents were given at least 48 hours to 'digest' this information before agreeing to 
participate. Within the interviews, confidentiality was maintained by anonymizing the information and ensuring privacy. The potential for coercion was avoided by the fact that the interviewer had no managerial authority over the research participants.

\section{Results and discussion}

\section{Preamble: the concept}

EBP was clearly a familiar concept to our respondents and they were able to offer definitions that were often sophisticated and nuanced, along with commentaries that attempted to set it in context. A dominant theme focused on the process of EBP and equating the concept with basing practice on research findings:

EBP means using all the valid research and evidence to inform practice.

A second theme involved placing the emphasis on clinical effectiveness and equating EBP with achieving favourable outcomes:

Adopting an EBP approach means you are able to say that something does or does not work, it's a common-sense approach to show that something is proven to work.

Opinion on the value of EBP was mixed. On the whole, however, most participants ascribed a positive value to EBP, sometimes as a means of justifying decision making and adding structure to practice:

I think EBP is a good way to 'back up' what I'm doing, I'm not always sure that what I do is evidence-based at the moment and it should be.

Using evidence in practice can give structure to your work.

Another dimension of this theme focused on the value of EBP in ensuring 'safe' practice:

It keeps people within safe boundaries of practice, it's an antidote to ritualistic old-fashioned practice.

On the other hand, some saw EBP as a 'fashionable, transitory concept':

I think it will end up being just another 'buzz phrase' and everyone's jumping on the bandwagon at the moment, there's a tendency for it to be empty rhetoric.

In addition, some espoused a more differentiated view of EBP, suggesting it can restrict or benefit practice depending on the practitioner's interpretation and the context of care:

EBP can be restrictive, but also productive, it can back you up as a practitioner. It doesn't have to stifle practice, it depends how you interpret and apply it.

Thus we can perhaps see some barriers to the wider implementation of EBP inherent in the definition used by practitioners. If the concept is defined solely in terms of the existing research literature, it is little wonder that it is difficult to apply to specific practice situations. Following the 
literature we have reviewed in the introduction, it would be easy to suggest that this is a matter of education - the participants should be enlightened as to the real nature and potential of EBP. However, we became aware that perhaps there was more at stake than this. Participants are not ignorant, but may even be strategically adopting a restricted definition of EBP so as to limit its intrusion into their practice and to justify their limited involvement with it, as we shall see later.

In line with a number of scholars of language in social life (Potter 1996, Gervais et al. 1999), we were intrigued by what was missing from these definitions. For example, while some writers have emphasized the importance of practitioners doing their own empirical research (French 1999), none of our participants mentioned this possibility. Moreover, participants explicitly excluded the use of clinical judgement where decisions concerning the optimal treatment are more arbitrary or where the results are less clear-cut, despite the fact that some authors on the subject see this as entirely compatible with the philosophy of EBP (American Medical Association 1992).

Therefore, the apparent divergence in views about the value of EBP was also a distinctly restricted one. What is striking is that whether or not they see it as a 'fashionable, transitory concept' or as a valuable basis for care, they concur in their exclusion of clinical judgement and personal research from the concept of EBP. This restriction is an important point, to which we shall return later in making sense of the role that EBP plays in participants' everyday work.

\section{Participants' construction of barriers to EBP}

With the concept defined, we then examined the transcripts for participants' accounts of barriers to the implementation of EBP. The definition adopted by practitioners is important in setting the stage for the kinds of barriers that participants can identify. We shall present these as personal barriers, professional and ethical barriers, organizational barriers, unfulfilled needs and the barriers that may emerge from training itself. These accounts of obstacles are not simply reflections of personal or organizational factors but are, we shall argue, carefully crafted not only to suit the definition of EBP to which they subscribe but also to enable them to manage its impact on their lives. As skilled managers of diversity, staff need to adjudicate between competing claims on their time, skills and resources. It may well be in their interests to keep parts of their practice, their thinking and their professional identity beyond the reach of EBP.

\section{Personal barriers}

This theme corresponds to the issues that are traditionally addressed in attempts to promote EBP. In this account, EBP is not possible because of the limitations of the participants themselves. As we have noted, the overwhelming assumption in the literature on EBP is that it is best promoted by educating the practitioner in the skills of seeking out, critically appraising and applying research to practice. Some of the respondents identified skill deficits in precisely this field:

Research is often boring and difficult to read and difficult to understand. 
Indeed, some also focused on education and the need for skill development:

I need skills in critically appraising research at a basic level, and, you know, how to search the literature properly.

These comments resonate with the skills taught in educational initiatives in evidence-based medicine. Indeed, some educators see the ideal situation as one where the practitioner's literature-searching skills are such as to make the technology 'transparent' to the process of seeking knowledge - this is one of the core skills proposed by Bilsker \& Gouldner (1999). Educators and practitioners are thus complicit in this deceptively simple story about the EBP enterprise. Together, they sustain the formulation that EBP can be achieved through the development of knowledge and skills. This is not solely a neutral account of educational needs, but one that practitioners can mobilize to explain why EBP has not penetrated their practice.

However, in the present study, the skills gap was only one of a complex array of factors that was constructed as inhibiting the progress of evidence-based care. Let us now examine a selection of these further barriers.

\section{Professional and ethical barriers}

One prominent set of issues that was seen to inhibit the development of EBP related to what was seen to be the traditions of the profession:

I think we've got a base on tradition really, I wouldn't say it was evidence-based. I think it's more passed down because it worked and this is what we've always done; whether there is actually evidence to support some practice, I'm not sure.

A second theme focused on the potential negative consequences of an over-reliance on research evidence in practice:

Experience counts for a lot and l'm not sure EBP values clinicians' experience.

Just basing practice on research evidence can be misleading and can lead people away from using their own judgement.

A further dimension of this theme was suggested by a participant who claimed that EBP could create a culture of 'prejudice towards nurses who had little interest in or knowledge of research'.

Here, then, practitioners are identifying other influences on their working lives that militate against the full-scale adoption of what they see as EBP. Having earlier ruled clinical experience out of the rubric of EBP, they are now able to counterpose the two and highlight the importance of the former. The restricted definition of EBP to which they subscribe thus allows them to foreground experiential clinical wisdom and maintain its privileged mystique. As one said:

The nurse-patient relationship is so complicated I don't think it is easy or even possible to research it, or even ethical. 
Thus, there are facets of the nursing process that will, in this view, be forever numinous; this serves to place these aspects beyond the gaze of EBP.

A third barrier to EBP was embedded within participants' notions of what it meant to be a nurse. This manifested itself in the form of a perceived conflict between the principles of EBP and user involvement:

There can definitely be conflict between what the client wants and what the evidence suggests that can make being totally evidence-based in what you do difficult.

Whereas exhortations to use an evidence-based approach in health care certainly foreground a sensitivity to the patient's emotional needs (American Medical Association 1992), in this participants' view there is - perhaps as a result of the restricted definition they adopted - a potential conflict between EBP and clients' wishes. Despite the inclusion of these as a factor in EBP in the literature, there is certainly far less guidance on how to resolve the dilemma.

The concern that there is something in the encounter between professional and client that is ineffable and cannot be easily researched has also bedevilled researchers who have tried to investigate psychological interventions (Parry 2000). In this sense, then, respondents are wrestling with some of the fundamental epistemological and methodological problems of the discipline. This barrier to EBP therefore arose from knowledge rather than ignorance.

A further professional barrier to the full-scale implementation of EBP related to what it meant to be a nurse working in community mental health. Respondents focused on the need to clarify what the core activities and role of the community mental health nurse should be from an evidence-based perspective. In line with the debate about nursing roles in the literature, a degree of role confusion is apparent on the face of things:

I think we need to get a handle on what our role is, I mean, what does the research say we should be doing? There seems to be so much disagreement.

A need to delineate core activities is indicated in one respondent's comment that:

We need to identify the core skills and then make sure that these are evidence-based.

The uncertainty about the skills, purpose and identity of nurses has been discussed in nursing for some time. This has been compounded by debates about the appropriate model and theory of mental health care, where a resurgence of interest in medical style theories and treatments has gained strength (Gournay 1995) at the same time as other authors have retained an insistence on seeing nursing in social and interpersonal terms (Forchuk 2001). Therefore, this uncertainty about what mental health nursing should involve reflects not a weakness that can be remedied by training but a sophistication as to the special features of the nursing process. Moreover, it is one which participants are quick to mobilize in accounting for the low priority attached to EBP in their work. As we have argued, this opens up spaces for the participants to keep their practice. Perhaps the 'ambiguity' and 'confusion' suggested here are not features that can be educated or debated out of nursing, but are 
essential features that allow the practitioners to tailor their practice to the circumstances.

\section{Organizational barriers}

The organizations within which nurses work may not make it easy for them to engage in EBP. The first organizational barrier was the issue of limited time to engage in EBP (Dunn et al. 1998): the participants were acutely aware of how the organizational constraints on their work limited their opportunity to gain information. Respondents often felt that:

We lack support really, you know, time- and resource-wise.

The second emergent theme highlighted the employing organization's culture as a further barrier. One respondent commented:

Other colleagues can be a barrier, like, not wanting to change or being resistive.

A number of participants commented that EBP-related activity might not be viewed as 'valid' work: I suppose one thing is that I don't feel it would be justifiable, you know, taking a day out to search for information.

Additionally, a further participant highlighted the perceived difficulty in prioritizing work: If you get a sudden influx of referrals, what comes first? Your education or your patients?

The third theme focused on the accessibility of research and contained numerous dimensions. The apparent lack of organizational support and available resources is highlighted in the following comment:

Accessibility is a major barrier, I struggle to access it. Within the work setting there's not much support, I tend to access it at home.

The problem of where and how to access research information is also identified:

It's something I find a bit off-putting, like having access to a library, you know, I mean I don't even know if I can have access to the university.

There was also an issue of distance from resources when working in the community: Most of the resources are in South Staffordshire, working in the community means I have to travel miles to get to it.

Information that is available often seems to be out-of-date.

There was also a perceived need for the administrative system to monitor and validate EBP activity:

There are no ... codes for activity related to EBP, it would help if we had codes to justify, I mean, you know if you spent all morning reading at the library.

Thus, some significant perceived barriers exist at the level of organization and administration. 
Whereas some accounts of evidence-based interventions in the literature stress how simple and quick it is to access evidence, these descriptions are often from teaching hospitals with networked computer terminals, subscriptions to electronic databases and well-stocked libraries on site (American Medical Association 1992), as well as clearly defined clinical problems. Clients often present with diffuse, but nevertheless disabling, problems in living that would necessitate extensive searching to evaluate - these facilities are not widely available to staff working in the community.

\section{Unfulfilled needs}

In this section, we have grouped together a number of themes that were deployed within participants' accounts of why evidence is not used in practice. They generally relate to the question of evidence not being in the right shape or form to apply in practice. Having previously formulated EBP as a good thing, the participants are then faced with the discursive task of explaining why they're not doing it. We have called this the discourse of unfulfilled needs, because they have in common the theme that certain facilities or activities are needed for the implementation of EBP but are absent, through no fault of the participants. The level of responsibility is identified as collective, organizational, bureaucratic or systemic.

Four main themes emerged around the perceived resources and support that were needed to develop EBP within community nursing. The first theme focused on the availability and accessibility of relevant, good-quality evidence.

Evidence-based information packages, on main practice issues, would have been a real help, you know, having the searching done for you.

Information, then, needs to be preselected and prepackaged in order to be usable. Other respondents also highlighted a need for contemporary, accessible data and for clarification of what all the research meant for practice:

We need a system for keeping information up to date and more accessible databases.

Clear practice guidelines, based on evidence, would help support practice.

In a sense, then, the desire among academic EBP enthusiasts to move away from a 'cookbook' approach to practice is being subverted here by the desire of practitioners to maintain that EBP requires a set of guidelines in order to be readily implemented.

Some respondents focused on the restrictions and ambiguity surrounding the availability of the practical resources needed to support EBP in community mental health settings:

We need more resources, like computer access and the Internet.

Easier access to libraries and the university would help, I don't think you can use the university databases unless you're on a course.

A further theme emerged around the importance of networking and sharing to develop a culture of EBP, and the implication that this was absent or impossible in community-based settings: 
Regular meetings, journal clubs or support groups to discuss EBP. Also seeing what goes on elsewhere and networking.

Some suggested that what was required was better dissemination of learning from academic courses:

I think we could use the work people are doing on courses more effectively, a lot of people are doing good work for assignments and degrees but you don't get to know about it always.

Thus, in accounting for the relatively low impact of EBP on their working lives, participants are showing that they are not merely 'ignorant Luddites' but are in fact keen to capitalize on the possibilities afforded by interacting with colleagues, using information technology and educational resources in becoming aware of ongoing research. Thus, by showing that they are receptive to EBP initiatives, participants are deflecting any possible criticism that they are merely idle, blinkered or are not taking responsibility for informing themselves.

In addition, some respondents suggested that a lack of experienced clinical supervisors meant that nurses were less likely to utilize new evidence-based interventions because of the lack of support and guidance. These comments highlight an important dimension of organizational culture. In training doctors in EBP, the importance of 'role models' has been highlighted (American Medical Association 1992), yet this is far less often foregrounded in nurse education.

\section{Training itself as a barrier to EBP?}

Whereas the general wisdom of the literature is that the practice of evidence-based health care can be taught, the present study disclosed the possibility that such teaching can even exacerbate the 'theory-skills' gap in current practice:

One of the difficulties I've got is that I recently did a course which was largely theoretical and academic, I didn't really come out with a skills base. So l've got evidence-based theoretical knowledge but lack the skills and confidence to put it into practice.

The drift of these and other related comments is that the available education itself seems to ghettoize EBP into something that one does in universities or on courses rather than a philosophy that pervades the organization as a whole and the individuals' practice within it. This quote highlights a paradoxical feature of educational initiatives - they may make the material more esoteric rather than more accessible. They may give practitioners an additional repertoire of reasons why EBP is not possible in their jobs.

\section{Discussion}

In making sense of the findings of the present study, it must be remembered that the sample of participants is small and geographically local to the UK Midlands, so any generalizations must be made with caution. However, our intention is not to make broad demographic claims about the 
generality of our findings but rather to use them to discern some clues about the issues that might be holding back the spread of EBP and suggest areas that might merit attention from policy makers, researchers, educators and those concerned to promote EBP.

As a result of our investigation our findings are twofold.

Firstly, participants are active in managing the contradiction between the overall ideology that EBP is a good thing vs. the fact that, to an outside observer, it may appear to be rarely in place in practice. Whereas we are not seeking to say that respondents are deliberately sabotaging EBP initiatives, we are suggesting that the staff interviewed here are adroit at deflecting the question of EBP and making it apparent that any deficits in their commitment to this way of working are either not their fault or alternatively one part of a principled objection.

This contradiction, we would argue, is not a weakness or a lack of theoretical autonomy or rigour on their part, but an important aspect of their discursive praxis in their working lives. They may be reluctant empiricists, but this forms part of an active management of their work and their accounts of themselves. There is a contradiction between the positive espousal of EBP on the part of most of them and the variety of practical constraints and missing desiderata that prevent their full-scale implementation of it. Their adoption of a restricted definition of EBP facilitated their construction of the restrictions on its use. EBP was always that little bit too far beyond what was achievable under the current circumstances. This pattern of findings echoes the situation discovered by Wetherell et al. (1987), who found that participants' espousal of equal opportunities rhetoric was undermined by their detailed account of the 'practical' and 'natural' constraints on women's emancipation. They called their participants 'unequal egalitarians' to highlight this contradiction. In parallel to this, we have adopted the term 'reluctant empiricists' for participants here.

In this way, our findings are reminiscent of the theory-practice gap that has often been complained of in nursing and other fields, such as education (Spouse 2001, Upton 1999). The research in the present study shows that, rather than simply being an effect of inadequate education or supervision (as has been suggested by some authors, e.g. Spouse 2001), the theory-practice gap might be carefully constructed by the practitioners as they go about their everyday working lives. Evidence-based praxis, then, does not involve a slavish adoption of EBP (Penney \& Warelow 1999).

The present study allows us - somewhat tentatively

- to offer a new way of thinking about the difficulties of implementing EBP. It is not, in our view, a difficulty that can be resolved by seeing the practitioners as somehow under-educated, under-skilled or information-deprived. Neither will we make much progress if we see them simply as Luddites seeking to delay the implementation of the new EBP regime. It might be possible to tackle this by adopting a bottom-up approach that validates what it is that community mental health nursing staff do with their time.

The second major strand in our findings echoes a good deal of the current literature. For example, the notion of limited time to engage in locating and utilizing evidence as well as limited resources and accessibility are findings echoed in many other studies (Lacey 1994, Funk et al. 1995, Veeramah 
1995, Black 1999, Retsas 2000), and in the present study we can see how these are related by participants to problematic organizational cultures, ethical and methodological issues and lack of accessibility. In addition, the kind of research that is often considered to be most valuable in health care, the randomized controlled double-blind clinical trial (Traynor 2000), is the most esoteric and difficult to apply to their own practice. Indeed, the experience of some of our participants in courses about EBP does not seem to have succeeded in tackling these difficulties, as they did not seem to have any strategy for dealing with these issues over and above those who had no such training.

As a result of our adoption of a qualitative method, we can see some features that have perhaps been underemphasized in questionnaire studies of barriers to EBP (Dunn et al. 1998, Parahoo 2000, Retsas 2000). We can see how the implicit concept of EBP itself to which practitioners subscribe excluding clinical experience or one's own research and relying on an often inaccessible technical literature - sets the scene for the limits they place on it in their own working lives. Moreover, we can see how their construction of their roles as nurses and their understanding of the research process makes it a difficult and potentially dehumanising - even unethical

- process to apply EBP to their work with clients. Indeed, this tendency seemed to be present in both those who had received additional training and those who had not, and seemed to be present in all the different age groups.

Therefore, the most important message from the present study is that we must be aware of the possibility that practitioners may be avoiding EBP not out of ignorance or lack of confidence but perhaps as a result of a principled view of the incompatibility of their day-to-day praxis with a mechanistic application of EBP methods. In this way, our findings echo an intimation from Traynor (1999) that there may be pockets of politicized resistance to the hegemonic 'ideology of evidence'. The limitations described in the present study - for example, skill, confidence and organizational constraints - are not just barriers, but represent a meticulously constructed and intelligently flexible set of strategies for limiting the impact of EBP upon practice. Those who wish to encourage the spread of EBP need to respect these sensitivities or they will risk alienating practitioners.

Whereas other authors have identified organizational factors as significant barriers, we have explored here the respondents' sense that time spent in reading and research is not catered for in their workplace, even down to minutiae such as the codes used to account for their time. This is especially so for community practitioners, who will often need to travel to access computer facilities and libraries to explore literature relating to their cases. Finally, we have the apparent paradox that education itself can sometimes make the activities of EBP appear even more remote and esoteric and give participants ever more sophisticated rationales for their reluctance to leap aboard the bandwagon.

Thus, whereas previous authors have highlighted the possibility that EBP might in practice be 'more rhetoric than reality' (Hicks 1998a, 1998b), we have gone further to identify the precise rhetorical strategies that can be deployed to maintain that state of affairs. In sum, it appears that despite the degree of support for EBP, it is defined in such a way that there are always 'good 
reasons' (Garfinkel 1967) in practical terms that prevent it being widely implemented.

From the point of view of policy makers and those keen to promote EBP, the message of hope is that respondents say they are willing to do a number of things that could support the cause of EBP. For example, some are keen to network and exploit the skills and activities of their colleagues, by means of meetings, and raise awareness of research done by colleagues (French 2000). If the focus on generating and finding evidence for practice is going to be more than an extra 'add-on' (Clarke \& Proctor 1999) to the work of CMHNs, then employing organizations must understand, promote and support a broad range of EBP activity. This becomes particularly important when one considers the complex environment of change and uncertainty within which CMHNs are required to work (Hopkinson et al. 1998).

In terms of what the participants do say they engage in to achieve the ideals of EBP, these strategies have some important limitations. For example, the finding that some describe themselves doing much of their reading and research at home, and have difficulty prioritizing EBP at work, is one of the factors that Hopkinson et al. (1998) say contributes to occupational stress. In addition, the multiple agendas for change represented by the National Service Framework and clinical governance initiatives can be bewildering. This, coupled with pressure to provide EBP without adequate support, might contribute further stress to an already stressed profession, and in so doing, inhibit appropriate change and improvement (ENB 1987). Thus, practitioners are able to paint a favourable picture of themselves as heroically struggling to implement some fragments of EBP at the expense of forfeiting their own leisure time. The reasons for the failure, in their formulation, do not originate in the staff themselves, but in the organization.

This difficulty in implementing EBP due to organizational constraints also places in context the desire of some practitioners for a latter-day 'cookbook' based on contemporary evidence of effectiveness. We could speculate that this discursive management of contradiction that we saw in participants' transcripts is a kind of strategy for managing this stress and excusing the implication that they should be doing more to include EBP in their daily work.

Effective implementation of EBP involves major time commitments on the part of practitioners and, more importantly, on the part of the institutions within which they work. The problems inherent in developing the facilities for EBP in healthcare organizations have also been highlighted by Donald (1998), who detected barriers such as access to computer facilities and even elementary issues like finding desk space, electrical sockets and telephone connections for the equipment introduced significant delays in project implementation. Generally, the evidence on which practice might be based was not easily accessible to the clinicians who needed it. Donald's project was based in hospitals; the problems of timely and convenient access are even more acute when it comes to practitioners working in the community. Whereas some information is accessible in the public domain via the Internet, much useful material requires subscriptions or university library membership. In addition, even these facilities often contain only the citation and the abstract of original literature and some material of most relevance to practitioners has limited information in the abstracts. Further 
research on EBP might profitably examine the kinds of uses that nurses are able to make of the kind of information that is available.

Our respondents have underscored the fact that to develop EBP within community mental health nursing, employing organizations must understand, promote and support EBP activity, and, just as importantly, the staff need to see that the organizations are doing so. In addition, research and EBP training initiatives need to be sensitive and 'tailored' to the unique issues and context of mental health nursing. EBP itself could usefully begin with evidence gathering on the part of those who seek to implement the policy. It requires a careful ethnographic observation of how this sort of activity might be fitted into nurses' daily work. It may be that rather than focusing on skills such as searching and evaluating the technical literature, it will have to be supplemented by training for practitioners in how to educate and transform their workplaces, as participants seem to feel entrapped by a culture that does not support EBP in practical ways.

Finally, there is also an expressed desire in the data to retain the intuitive mystery of the therapeutic process that is bound up with notions of professional purpose and ethics. If practitioners are to retain a sense of their own integrity and also adopt a more self-reliant role as evidence-based therapists, then it is important that their overly restrictive notions of EBP be challenged and broadened. It could be emphasized that EBP can indeed include their own experience as a critical device for interrogating both the literature and their employing organizations themselves. It is in this way that the profession will begin to be more than reluctant empiricists and will be enabled to include ever more evidence in their daily praxis.

\section{References}

American Medical Association (1992) Evidence-based medicine: a new approach to teaching the practice of medicine. Journal of the American Medical Association 268 (17), 2420- 2425.

Barker P.J. (2001) The tidal model: the lived experience in person-centred mental health nursing care. Nursing Philosophy 2, 213-223.

Berk M. \& Leigh Janet M. (1999) Evidence-based practice: doctrine or trap? Journal of Evaluation in Clinical Practice 5 (2), 149-152.

Bilsker D. \& Gouldner E.M. (1999) Teaching evidence-based practice in mental health.

Evidence-based Mental Health 2 (3), 68-69.

Black S. (1999) Not enough time to implement research. Nursing Standard 13 (33), 8.

Bonell C. (1999) Evidence-based nursing: a stereotyped view of quantitative and experimental research could work against professional autonomy and authority. Journal of Advanced Nursing $\mathbf{3 0}$ (1), 18-23.

Brooker C. (2000) A decade of evidence-based training for work with people with serious mental health problems: progress in the development of psychosocial interventions. Journal of Mental Health 10 (1), 17-31.

Brooker C., Repper J.M. \& Booth A. (1996) The effectiveness of community mental health nursing: a 
review. Journal of Clinical Effectiveness 1 (2), 44-50.

Clarke L. (2000) Opinion: the value of nursing research. Mental Health Practice 4 (3), 12-13.

Clarke C. \& Proctor S. (1999) Practice development: ambiguity in research and practice. Journal of Advanced Nursing 30 (4), 975-982.

Closs S. \& Cheater F. (1999) Evidence for nursing practice: a clarification of the issues. Journal of Advanced Nursing 30 (1), 10-17.

Colaizzi P.F. (1978) Psychological research as the phenomenologist views it. In: Existential Phenomenology for Psychology (Eds R. Valle \& M. King), pp. 48-71. Oxford University Press, New York.

Cormack D.F.S. (1996) The Research Process in Nursing, 3rd edn. Blackwell Scientific Publications, Oxford.

Coyler H. \& Kamath P. (1999) Evidence-based practice. A philosophical and political analysis: some matters for consideration by professional practitioners. Journal of Advanced Nursing 29 (1), 188-193.

Department of Health (1999a) The Effect of Evidence-based Practice on General Health Policy Measures. Department of Health, London.

Department of Health (1999b) The National Service Framework for Mental Health. Department of Health, London.

Dinsdale P. (2000) Robust research is key to nurses' political influence. Nursing Standard 14 (31), 7. Donald A. (1998) The Front Line Evidence-based Medicine Project. NHS Executive, North Thames Regional Office Research and Development, London.

Dunn V., Crichton N., Roe B., Seers K. \& Williams K. (1998) Using research for practice: a UK experience of the Barriers scale. Journal of Advanced Nursing 27, 1203-1210.

ENB (1987) Managing Change in Nursing Education. City Print Ltd, Milton Keynes.

Flaming D. (2001) Using phronesis instead of research-based practice as the guiding light for nursing practice. Nursing Philosophy 2, 251-258.

Forchuk C. (2001) Evidence-based psychiatric/mental health nursing. Evidence-based Mental Health 4, 39-40.

French P. (1999) The development of evidence-based nursing. Journal of Advanced Nursing 29 (1), 72-78.

French B. (2000) Networking for research dissemination: collaboration and mentorship. Nurse Researcher 7 (3), 13-

21.

Funk S.G., Champagne M.T., Tornquist E.M. \& Wiese R.A. (1995) Administrators' views on barriers to research utilization. Applied Nursing Research 8, 44-49.

Garfinkel H. (1967) Studies in Ethnomethodology. Prentice Hall, New York.

Gervais M.-C., Merant N. \& Penn G. (1999) Making sense of absence: towards a typology of absence in social representations theory and research. Journal for the Theory of Social Behaviour 29 (4), 
419-444.

Ghali W.A., Saitz R., Sargious P.M. \& Hershman W.Y. (1999) Evidence-based medicine and the real world: understanding the controversy. Journal of Evaluation in Clinical Practice 5 (2), 133-138.

Gournay K. (1995) What to do with nursing theories. Journal of Psychiatric and Mental Health Nursing 2 (5), 325-327.

Grant A. \& Mills J. (2000) The great going nowhere show: structural power and mental health nurses. Mental Health Practice 4 (3), 14-16.

Hawes L. (1977) Towards a hermeneutic phenomenology of communication. Communication Quarterly 25, 30-41.

Hicks C. (1998a) Barriers to evidence-based care in nursing: historical legacies and conflicting cultures. Health Services Management Research 11, 137-147.

Hicks C. (1998b) Evidence-based care in nursing: reforms versus research, rhetoric versus reality. Health Services Management Research 11, 246-254.

Holloway F. (2000) Mental health policy, fashion and evidence-based practice. Psychiatric Bulletin 24, 161-162.

Hopkinson P., Carson J., Brown D., Fagin L., Bartlett H. \& Leary J. (1998) Occupational stress and community mental health nursing: what CPNs really said. Journal of Advanced Nursing 27 (4), 707-712.

Hunt M. (1987) The process of translating research findings into practice. Journal of Advanced Nursing 12, 101-110.

Jackson W. (1999) Psychosocial interventions as apart of a mental health Project 2000 branch programme. Mental Health Practice 3 (2), 16-17.

Lacey E.A. (1994) Research utilization in nursing practice: a pilot study. Journal of Advanced Nursing 19, 987-995.

Lines K. (2001) A philosophical analysis of evidence-based practice in mental health nursing.

Australian and New Zealand Journal of Mental Health Nursing 10, 167-175.

McKenna H., Cutcliffe J. \& McKenna P. (2000) Evidence-based practice: Demolishing some myths.

Nursing Standard 14 (16), 39-42.

McMillan I. (2000) Reappropriating madness. Mental Health Practice 3 (4), 8-9. Mitchell

G. (1997) Questioning evidence-based practice for nurses. Nursing Science Quarterly $10,4$.

Morgan D.C. \& Krueger R.A. (1997) The Focus Groups Kit. Sage Publications Inc., London.

NHS Centre for Reviews and Dissemination (1999) Getting evidence into Practice. University of York and Royal Society of Medicine, York.

Nieboer R., Moss D. \& Partridge K. (2000) A great servant but a poor master: a critical look at the rhetoric of evidence-based practice. Clinical Psychology Forum 136, 17-19.

Parahoo K. (2000) Barriers to, and facilitators of, research utilization among nurses in Northern Ireland. Journal of Advanced Nursing 31 (1), 89-98. 
Parry G. (2000) Evidence-based psychotherapy: special case or special pleading? Evidence-based Mental Health 3, 35-37.

Pearcey P.A. (1995) Achieving research-based nursing practice. Journal of Advanced Nursing 22 , 33-39.

Penney W. \& Warelow P.J. (1999) Understanding the prattle of praxis. Nursing Inquiry 6, 254-268.

Polit D. \& Hungler B. (1995) Nursing Research. JB Lippincott, Philadelphia, PA.

Potter J. (1996) Representing Reality. Sage Publications Inc., London.

Potter J. \& Wetherell M. (1987) Discourse and Social Psychology: Beyond Attitudes and Behaviour.

Sage Publications Inc., London.

Ray L. (1999) Evidence and outcomes: agendas, pre-suppositions and power. Journal of Advanced Nursing 30 (5), 1017-1026.

Retsas A. (2000) Barriers to using research evidence in nursing practice. Journal of Advanced Nursing 31 (3), 599-606.

Reynolds S. (2000) Evidence-based practice and psychotherapy research. Journal of Mental Health 9 (3), 257-266.

Sackett D.L., Richardson W.S., Rosenberg W. \& Haynes R.B. (1997) Evidence-based Medicine: How to Practice and Teach EBM, Churchill Livingstone, New York.

Schon D. (1987) Educating the Reflective Practitioner. Jossey Bass, London.

Slevin E. \& Sines D. (2000) Enhancing the truthfulness, consistency and transferability of a qualitative study: using a manifold of approaches. Nurse Researcher 7 (2), 79-95.

Spouse J. (2001) Bridging theory and practice in the supervisory relationship: a sociocultural perspective. Journal of Advanced Nursing 33 (4), 512-522.

Thompson D. (1998) Why evidence-based nursing? Nursing Standard 13 (9), 58-59.

Traynor M. (1999) The problem of dissemination: evidence and ideology. Nursing Inquiry 6, 187-197. Traynor M. (2000) Purity, conversion and the evidence-based movements. Health 4 (2), 139-158. Upton D.J. (1999) How can we have evidence-based practice if we have a theory-practice gap in nursing today? Journal of Advanced Nursing 29 (3), 549-555.

Veeramah V. (1995) A study to identify the attitudes and needs of qualified staff concerning the use of research findings in clinical practice within mental health care settings. Journal of Advanced Nursing 22 (5), 855-861.

Walsh M. \& Ford P. (1989) Nursing Rituals: Research and Rational Actions. Butterworth Heinemann, Oxford.

Wetherell M., Stiven H. \& Potter J. (1987) Unequal egalitarianism: a preliminary study of discourses concerning gender and employment opportunities. British Journal of Social Psychology 26 (1), 59-71.

Wilson-Barnett J., Corner J. \& DeCarle B. (1990) Integrating nursing research and practice - the role of the researcher as teacher. Journal of Advanced Nursing 15, 154-167. 